\section{AD VALOREM}

Journal of Young Researchers

\section{Acceso $\mathbf{0}$ abierto}

Para citaciones: Valencia, V., Rodríguez, T., y Rincón, M. (2021). ISO 14001 una alternativa de gestión ambiental en las empresas colombianas a favor de la calidad del agua, Años 2013-2019. Revista de jóvenes investigadores Ad Valorem, 4(1), 732.

Editor: Bernardo Romero Torres. Universidad de Cartagena-Colombia.

\section{Tipología IBN Publindex:} Artículo de investigación científica y tecnológica

ARTÍCULO DE INVESTIGACIÓN

\title{
ISO 14001 una alternativa de gestión ambiental en las empresas colombianas a favor de la calidad del agua, 2013-2019
}

\author{
Valentina Valencia Marin' ${ }^{1}$, Tania Rodríguez Cardona ${ }^{2}$, Miguel Ángel Rincón Taborda ${ }^{3}$ \\ ${ }^{1}$ Universidad del Quindío - Colombia \\ ${ }^{2}$ Gestión de Ingresos, CRQ, Armenia, Quindío, Colombia \\ ${ }^{3}$ Universidad del Quindío - Colombia
}

\section{RESUMEN}

El siguiente artículo presenta el análisis de la certificación de la norma ISO 14001 como un sistema de gestión ambiental que adoptan las empresas y las implicaciones positivas o negativas que esta trae para el capital natural agua. A partir de ello, se identifican y analizan los datos entre los años 2013 a 2019 en función de características empresariales y ambientales que involucran al capital natural agua, a partir de un estudio sobre la calidad de esta. Mediante un modelo de datos panel bajo la metodología de corte transversal y series temporales se determina el impacto, tomando como variable dependiente la calidad del agua, dado que explica el capital natural agua entorno a la gestión ambiental. Como resultado, se encuentra que la certificación en la norma ISO 14001 posee una relación positiva de baja significancia con la calidad del agua, la cual es explicada principalmente por la baja motivación de las empresas a certificarse. Posteriormente, la investigación muestra la importancia de los sistemas de gestión ambiental, además de establecer diferentes relaciones entre la certificación, la competitividad y las distintas afectaciones al capital natural. Finalmente, se obtuvieron resultados relacionados con el ambiente y los comportamientos negativos que genera la industria dentro de sus sistemas de producción que fueron comparados a través de las mismas variables de estudio.

Palabras clave: Calidad del agua; Capital natural agua; Colombia; Empresas; ISO 14001; Sistemas de gestión ambiental.

\section{ISO 14001 an environmental management alternative in Colombian companies in favor of water quality, 2013-2019}

Copyright: (C) 2021. Valencia, V., Rodríguez, T., y Rincón, M. Este es un artículo de acceso abierto, distribuido bajo los términos de la licencia

https://creativecommons.org/licenses/by-

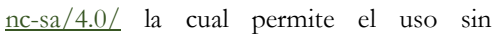
restricciones, distribución y reproducción en cualquier medio, siempre y cuando que el original, el autor y la fuente sean acreditados.

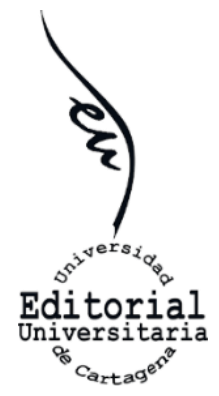

\section{ABSTRACT}

The following article presents the analysis of ISO 14001 certification analysis as an environmental management system adopted by companies and the positive or negative implications that it brings for natural capital water. From this, the data between the years 2013 and 2019 are identified and analyzed based on business and environmental characteristics that involve the natural water capital, based on a study on water quality. Through a panel data model under the cross-sectional and time series methodology the

'Estudiante $9^{\circ}$ semestre de Economía, Universidad del Quindío; Facultad de Ciencias Económicas y Administrativas. Armenia, Quindío, Colombia. Correo Electrónico: vvalenciam_2@uqvirtual.edu.co

2Economista, Universidad del Quindío; Contratista Gestión de Ingresos, CRQ, Armenia, Quindío, Colombia. Correo electrónico: Trodriguezc@uqvirtual.edu.co

${ }^{3}$ Estudiante $9^{\circ}$ semestre de Economía, Universidad del Quindío; Facultad de Ciencias Económicas y Administrativas. Armenia, Quindío, Colombia. Correo Electrónico: marincont@uqvirtual.edu.co 
impact is determined, taking water quality as a dependent variable, since it explains the natural capital water around environmental management. As a result, it is found that the certification in ISO 14001 standard has a positive relationship of low significance with the quality of the water, which is mainly explained by the low motivation of companies to be certified. Subsequently, the research shows the importance of environmental management systems, in addition to establishing different relationships between certification, competitiveness and the different impacts on natural capital. Finally, results related to the environment and negative behaviors generated by the industry within its production systems were obtained, which were compared through the same variables of the study.

Keywords: Companies; Colombia; Environmental management systems; ISO 14001; Natural capital water; Water quality.

\section{INTRODUCCIÓN}

La implementación de planes de gestión ambiental en el proceso empresarial son un tema de vital importancia para la conservación de los capitales naturales, dado que las empresas en sus sistemas de producción demandan una gran cantidad de bienes y servicios ecosistémicos, lo cual provoca un deterioro del medio ambiente, afectando así; el agua, el aire, el suelo y las especies que conviven en el medio natural. En este sentido, Gonzáles (2015) afirma que solo el 10\% de la oferta hídrica está destinada para el abastecimiento humano mientras que el 90\% restante es demandado por sectores como la agricultura y la industria. De esta manera, las empresas como plantea Zapata (2007), son consideradas "sistemas socio-económicos, tecnológicos y antiecológicos" (p.12), las cuales siempre tienen por objetivo maximizar sus ganancias sin importar todo el daño que estas pueden generar.

En ese orden de ideas, Cardona (2020) establece que Colombia se encuentra entre los primeros 5 países más ricos en recursos naturales, el cual posee el 10\% de la biodiversidad del planeta, contando con 1.1 millones de $\mathrm{km} 2$ y con un total de 1'214.258 Mm3/año en cuanto a fuentes hídricas, no obstante, un alto porcentaje de este recurso hídrico se está viendo perjudicado por el manejo inadecuado de las empresas. Del mismo modo, La Organización de las Naciones Unidas para la Educación, la Ciencia y la Cultura (UNESCO, 2016) "calcula que tres de cada cuatro empleos en el mundo dependen en mayor o menor medida del agua", de manera que este recurso se considera el motor del crecimiento económico.

Por otro lado, una prueba del mal manejo del recurso hídrico en las empresas se evidencia mediante el Índice de calidad de agua expuesto por el Instituto de Hidrología, Meteorología y Estudios Ambientales (IDEAM, 2019), el cual encontró que la calidad de este recurso varia entre aceptable y muy malo (Figura 1). Además, dentro de este seguimiento no se encontró el recurso hídrico dentro de 
la categoría "buena" para ninguna estación de monitoreo. A su vez, se destaca que menos del 50\% de las aguas residuales reciben un tratamiento de calidad, y que un 32\% de los municipios de Colombia no cuentan con acceso a agua potable, lo que hace de vital importancia investigaciones sobre el recurso hídrico.

Mapa 1. Índice de calidad de agua, Año 2016.

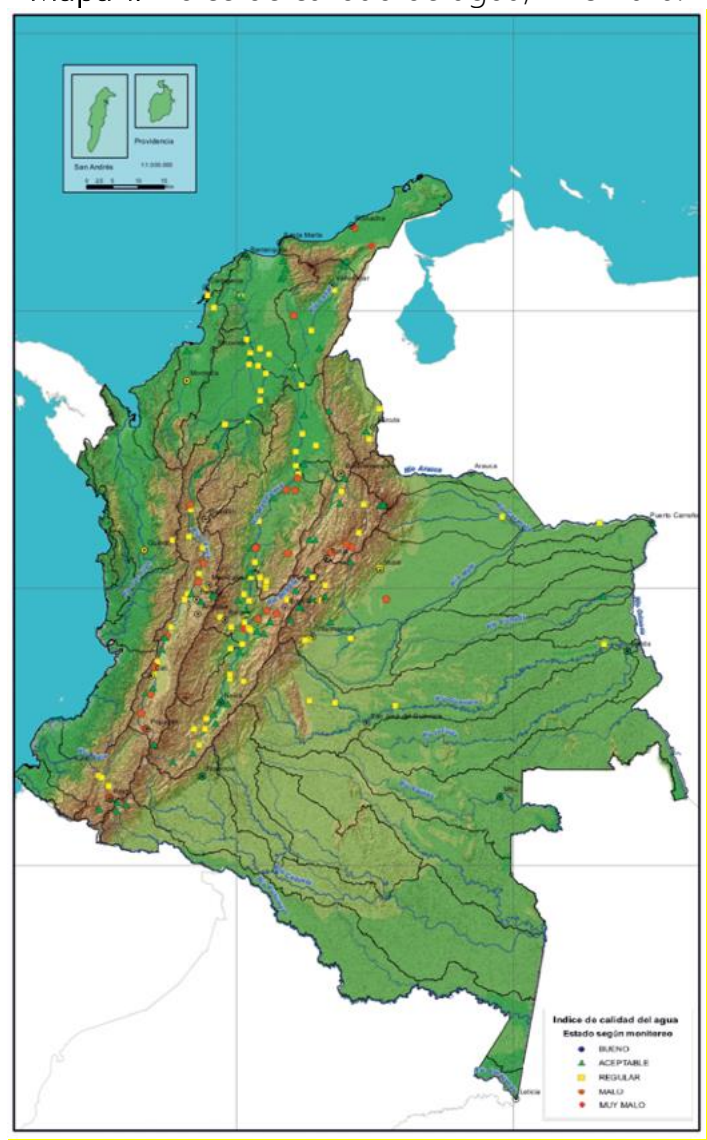

Fuente: Tomado del Estudio Nacional del Agua 2018 (p.242), por IDEAM, 2019.

Asi mismo, el estudio nacional de aguas realizado por el IDEAM (2018), determina las principales causas asociadas a la disminución en la calidad de agua y en la oferta de esta. Lo mencionado anteriormente, se debe a la débil estructuración de un sistema basado en los recursos naturales y también a actividades como la agricultura mediante el uso de agroquímicos, generando erosión de suelos; la minería (oro y plata) mediante el vertimiento de mercurio al suelo y al agua; el uso de sustancias químicas para la transformación de coca y por ultimo, actividades industriales tales como la deforestación, la acumulación de sedimentos, etc.

Para mitigar los impactos negativos por parte de las empresas, surgió el concepto de gestión ambiental, el cual permite un equilibrio entre el desarrollo de la población, el crecimiento económico y el medio ambiente. Según el Instituto 
Colombiano de Normas Técnicas y Certificación (ICONTEC, 2015), los modelos de gestión ambiental permiten mitigar los efectos causados por las empresas en el desarrollo de sus actividades productivas, lo que supone una serie de recomendaciones para reorientar sus procesos internos y el relacionamiento con los grupos de interés. A su vez, Porter en 1990 aseguraba que las regulaciones ambientales generaban un impacto sustancial y positivo en la competitividad de las empresas y la economía en función competitiva para el mercado. Dicho autor establecía que "Las regulaciones por parte de los entes gubernamentales de manera estricta generan ventajas competitivas, dado que un estándar estricto orienta el rendimiento de un buen producto y el impacto ambiental obligando a las empresas a alcanzar calidad" (Panayotou y Vincent, 1999, p.26). Para Porter la maximización de las ganancias y disminución en los estándares de contaminación se complementan en el uso eficiente de los recursos, administración de calidad total y la innovación en tecnologías de punta.

En la actualidad, se ha dado un aumento significativo en los sistemas de gestión ambiental en Colombia, aunque su implementación no ha sido tan predominante en las empresas. Para el año 2019, se tiene que 3.071 empresas estaban certificadas en la ISO 14001 (Figura 1), lo cual representa tan sólo un 0,001\% del total de empresas colombianas, dejando una cifra de 1'640.778 empresas sin certificar (Figura 2). Estas cifras permiten cuestionar el interés por parte de las empresas en mejorar su relación con el medio ambiente, y la efectividad de la norma ISO 14001 para la conservación del capital, dado que, a pesar de que las empresas en los últimos años se han acogido a estos cambios aún se sigue evidenciando grandes problemáticas en el cuidado del recurso hídrico.

Figura 1. Total de Empresas Certificadas en la ISO 14001 en Colombia, 2013-2019.

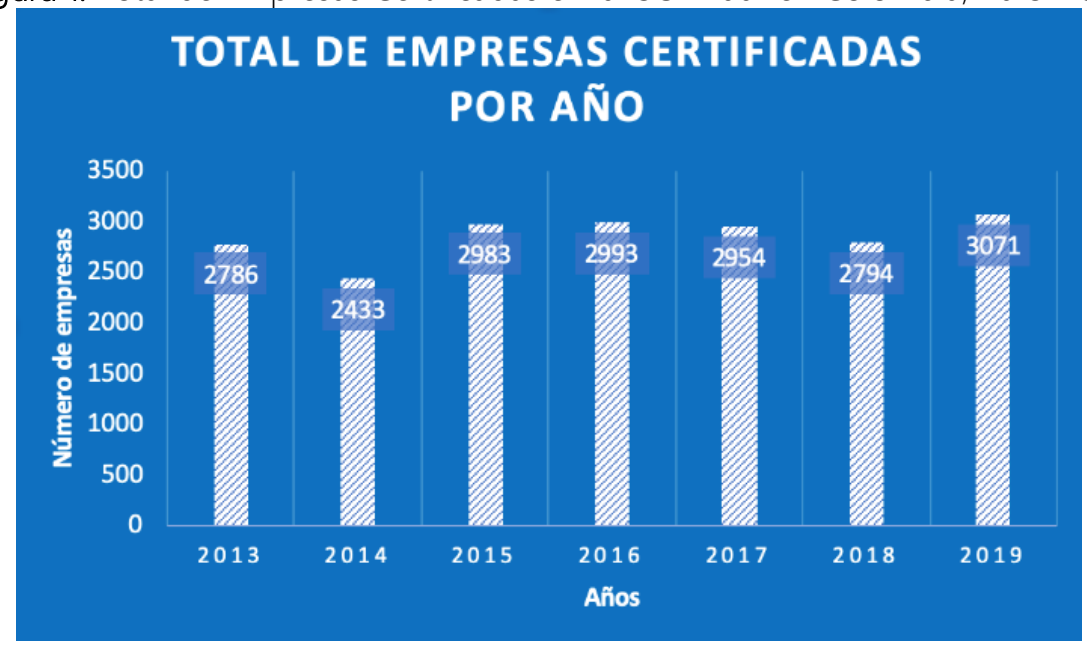

Fuente: Elaboración propia. Con datos CEPALSTAT 2019. 
Figura 2. Total de Empresas no Certificadas en la ISO 14001 en Colombia, 2013-2019.

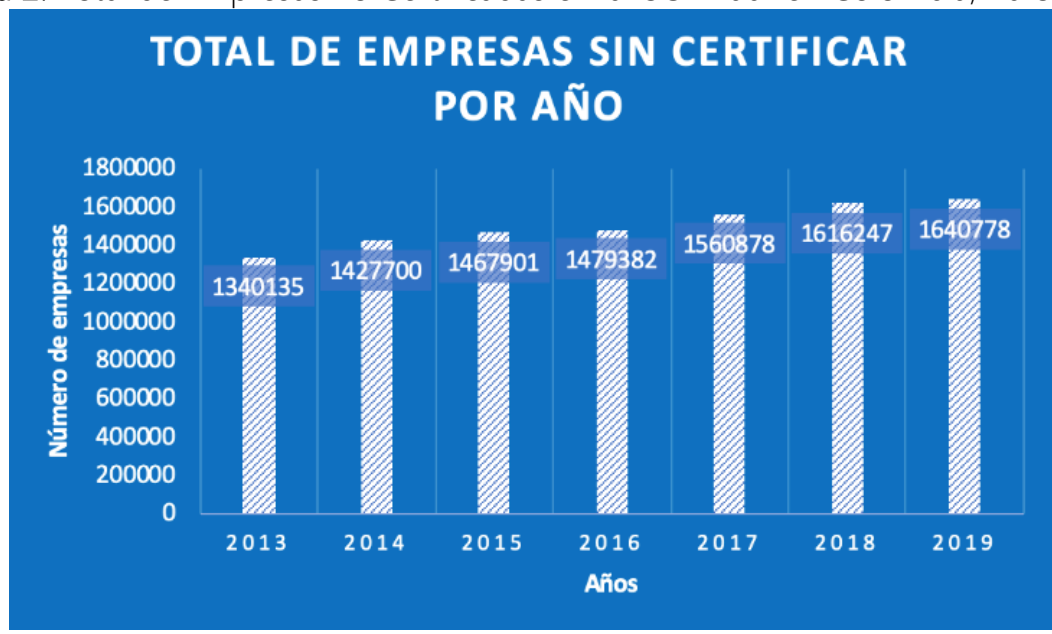

Fuente: Elaboración propia. Con datos Confecámaras 2020.

La presente investigación tiene por objetivo analizar la certificación en la Norma de gestión ambiental ISO 14001 por parte de las empresas colombianas y las implicaciones positivas o negativas que esta alternativa trae al cuidado de la calidad del recurso hídrico, entre los años 2013 a 2019, respondiendo a la falta de investigaciones bajo los parámetros de estudios de las normas de gestión ISO y teniendo en cuenta que uno de los componentes ambientales de mayor uso por parte de las empresas es el agua. Esta norma es una herramienta útil para la industria, permitiéndoles mejorar su relación con el medio natural, generándoles calidad en los productos y competitividad en el mercado.

Esta investigación se basa en la existencia de una relación directa entre las empresas como agentes contaminantes del capital natural y el agua como parte de la materia prima de la producción empresarial, dicha situación se especifica en cuatro instancias y están descritas de la siguiente manera; en primera instancia se realizó una revisión de literatura alrededor del tema de estudio y los aportes de diferentes autores. En segunda instancia se estableció la relación de las empresas y sus características organizativas con el capital natural agua a través de su calidad, mediante una metodología de tipo exploratorio con un enfoque cuantitativo de corte econométrico. En tercera instancia se identifican los departamentos que presentan un mayor o menor desempeño ambiental y empresarial en términos de la conservación del capital natural agua, con el fin de descubrir si esta norma logra mitigar los impactos negativos en la calidad del agua, de tal modo que se reconozca la necesidad de conservar el capital natural agua y los servicios ecosistémicos que esta provee. Por última instancia se desarrollaron una serie de recomendaciones en materia de gestión ambiental territorial y empresarial para la conservación del capital natural agua. 


\section{REVISIÓN DE LITERATURA}

La literatura relacionada con la aplicación y certificación de la ISO 14001 es interdisciplinar y amplia, sin embargo, los estudios encontrados no se enfocan directamente en el impacto y la eficiencia que ha tenido la implementación de la Norma ISO 14001 en las empresas para la conservación del capital natural, sino que se centran en la implementación de la norma en una empresa o sector específico con el fin de reorganizar sus procesos productivos y poder reducir los niveles de contaminación que estos generan sobre el medio ambiente. La presente revisión bibliográfica se aborda a partir del origen de la realización del estudio, organizado por orden cronológico y abordando casos a nivel nacional e internacional.

En estudios nacionales como es el caso de la zona oriental colombiana de Enríquez (2009), se observa un conjunto de estrategias para la implementación de la Norma ISO 14001 en empresas productoras de palma de aceite, dado que dicha actividad es altamente contaminante y produce pérdida de fauna y flora, contaminación atmosférica y contaminación de fuentes hídricas debido a vertimientos y emisiones atmosféricas. Mediante dicha investigación, se analizó el proceso productivo de estas, para luego identificar los impactos negativos que ocasiona en el medio ambiente. Para ello, realizan un análisis de tipo cuantitativo y una evaluación de la población de palma de Aceite en los departamentos del Meta y Casanare con el fin de proponer una política ambiental y una propuesta metodológica que permita optimizar los procesos y hacer de los mismos más rentables al reducir costos. De esta manera, han podido establecer procedimientos que les permitan cumplir con los requisitos de la norma ISO 14001, reduciendo la contaminación por parte de los procesos productivos de plantación y el procesamiento de palma.

Por su parte, Páez (2017) estudia el aporte y la implementación de la gestión ambiental en las pequeñas, medianas y grandes empresas colombianas que pertenecen al sector servicios con el fin de que este estudio permita proteger el medio ambiente y contribuya a la productividad y competitividad de las empresas. Para ello, se tomaron empresas que contaran con componentes de gestión ambiental como la ISO 14001, tales como Empresas Públicas de Medellín, la empresa Manpower Group en Argentina y por último la empresa Aguas de Cartagena. Esta última, ha tenido una buena gestión ambiental y también una buena gestión financiera. Dicha empresa, se encarga de garantizar la disponibilidad de agua para todos sus usuarios, además de luchar contra la pobreza extrema, también cuenta con diferentes proyectos de gestión ambiental como ha sido el control de olores y vertidos, la protección de zonas verdes que han permitido la reforestación de 2,2 hectáreas y la recuperación de 341.482m2 
zonas verdes, resaltando los impactos de una buena gestión ambiental para la conservación del medio ambiente.

En ese mismo sentido, la Influencia de los Sistemas de Gestión Ambiental ISO 14001 en las organizaciones, quedó definida mediante una investigación realizada en empresas manufactureras de Barranquilla en el año 2017. En este estudio, se analizó un grupo de trece empresas certificadas por el instituto colombiano de normas técnicas -Icontec-, teniendo como objetivo identificar los beneficios de la aplicación de sistemas de gestión ambiental para el crecimiento rentable empresarial. Para ello, se desarrolló una investigación de metodología exploratoria mediante el uso de encuestas. Con esto, encontraron que la implementación de la norma tiene un mayor impacto en la gestión organizacional y en el aprovechamiento de recursos, por el esquema del sistema de gestión ambiental, donde las empresas revelan una reducción en el consumo de materias primas y un mejor uso o reciclado de residuos sólidos. Esto les permite reducir sus impactos al medio ambiente y proponer estrategias para la gestión, destacando entre estas el proyectar inversiones en el área ambiental, con lo cual las empresas retribuirían en algo al medio ambiente (Acuña et al.,2017). A partir de esta investigación, se observa que la norma ISO 14001 es un medio para mejorar la competitividad, aunque no se hace énfasis en el tema de verdadera importancia como lo es el impacto medio ambiental. Sin embargo, se manifiesta el avance que cumple la norma sobre el sistema de gestión de residuos sólidos, con lo que se incentiva aún más a la exploración de la investigación en su impacto sobre su relación con el capital natural.

Vargas (2019) resalta a la ISO 14001 como un sistema de gestión ambiental que posibilita el cumplimiento de los Objetivos del Desarrollo Sostenible (ODS), dejando clara su relación con el medio ambiente. Para dicha investigación, Vargas se enfocó en cuatro empresas, dos de Colombia y dos de Brasil: Argos S.A, Energía del Quindío S.A ESP, Eucatex y Petrobras, que adoptaron la norma ISO 14001 y en las cuales se resalta el uso y el procesamiento adecuado de los residuos sólidos generados para limitar así su impacto ambiental, a través de medidas de reutilización, reciclaje y adecuada gestión de tratamiento. Además, empresas como Eucatex y Energía del Quindío S.A ESP, tenían entre sus planes de gestión ambiental la siembra de árboles para la preservación del medio ambiente. Finalmente, el trabajo resalta que la implementación de la norma propicia a generar mejores políticas que cuiden el medio ambiente y que la certificación no sólo ayuda a la organización sino también al país que las engloba permitiendo el alcance de nuevos mercados y el cumplimiento de los ODS.

Finalmente, se toma el trabajo de Ceballos, Ramírez y Salazar (2019), que tiene por objeto establecer la importancia de la implementación de la ISO 14001 en las organizaciones. Para ello, estudian las funciones y las responsabilidades que 
deben de tener las distintas organizaciones para que la implementación de la norma sea posible. Por otro lado, establecen que los impactos ambientales de la implementación de la norma pueden conducir a minimizar el uso del agua, reducir la degradación ambiental, conservar los recursos, valorar los impactos ambientales mediante el ciclo de vida del producto y finalmente reducir los accidentes medioambientales. Respecto a los impactos económicos se tiene que la implementación tiende a lograr impactos positivos en la competitividad, maximización de la producción y mayor rentabilidad en el largo plazo. Con lo anterior se hace énfasis en la importancia de la implementación de la norma y resalta el interés por definir la relación que tienen las empresas certificadas en la ISO 14001 y como esta ayuda a la conservación del capital natural. Por último, a pesar de que esta investigación revela los impactos positivos de la norma, no trabaja con datos numéricos, ni se establecen sus impactos de manera clara sobre el capital natural.

De la revisión de literatura anterior se puede decir que este tipo de certificación ocasiona un cambio estructural en las empresas, lo cual puede traducirse en beneficios para la empresa y para el ambiente natural.

\section{METODOLOGÍA}

Este trabajo parte de una línea de tipo exploratorio, en donde se analiza e investiga un tema que aún no ha sido estudiado en absoluto o no ha sido analizado a gran profundidad, como lo es la Norma ISO 14001 y su efectividad (Castillero, 2017). Por tal razón, se involucraron investigaciones similares para comprender de manera macroeconómica como ha funcionado la Norma ISO en lo que respecta al capital natural y la función de la empresa en su gestión ambiental entre los años 2013 a 2019. Posteriormente se agrupo la información estadística, con la diferenciación de bases de datos constituidas por tipologías empresariales y ambientales. Siguiente a esto, se le dio enfoque principal al capital natural agua mediante la calidad de este.

Por otra parte, el enfoque que se aplicó a la investigación es cuantitativo, dado que la investigación consiste en el estudio y análisis de la realidad mediante el uso de datos numéricos a través de herramientas de carácter estadístico y econométrico con el fin de verificar o refutar una hipótesis (Otero, 2018). Como modelo econométrico se diseñó un modelo estadístico por medio de un panel con datos de corte transversal y series de tiempo en el que se incluyeron características empresariales, tales como la competitividad, la densidad empresarial departamental, el tamaño del mercado, empresas certificadas en la ISO 14001 y no certificadas y componentes ambientales como la proporción de superficie cubierta por bosque y la tasa de afectación de desastres naturales para la conformación total del panel. Por otro lado, la serie de tiempo; esta 
dimensionada en un periodo de siete años (2013-2019), donde se busca recibir un impacto de forma negativa o positiva de los datos delimitados.

\subsection{Estrategia econométrica}

En los últimos años se ha resaltado la importancia en mezcla de datos para obtener información de calidad y detallada, sugiriéndose unión de los datos de corte transversal como la obtención de una o varias variables de información diferente pero en un determinado periodo de tiempo, que para este caso se fundamenta en la información de la base de datos del Consejo Privado de Competitividad (CPC) y por otro lado, la información de las series de tiempo que indican el conjunto de observaciones sobre los valores de una variables en diferentes tiempos, es claro que la información para este tipo de datos se debe recolectar en clasificaciones regulares y no mezcladas, es decir, toda la información que se va agrupar debe tener la forma diaria, semanal, mensual, semestral o anual pero nunca mezclada, dado que se incurriría en una incubación y mezcla inexplicada para el modelo econométrico. (Gujarati \& Porter, 2009)

En econometría es importante entender la procedencia de los datos, para así elaborar la búsqueda del mejor ajuste de datos explicativos hacia la variable dependiente. Para concluir lo dicho en el párrafo anterior, los datos de corte transversal aluden a datos individuales de un estudio en el cual no se involucra la información temporal, pero si la dimensión estructural o el porqué de la base de datos que se está escogiendo, los datos por series de tiempo son la introducción de variables al software de modelación por detalle unitario con un periodo de tiempo usualmente igual, lo que terminaría siendo datos de dimensión temporal. (Gujarati \& Porter, 2009)

Como descripción económica de un análisis completo de datos se pretende;

$$
Y=\left(i=1, \ldots, N_{t}=1, \ldots, T_{k}=1, \ldots, K\right)(1)
$$

Fuente: Elaboración en base a información de (Gujarati y Porter, 2009)

Donde:

\section{$\beta_{0}, \beta_{k}$}

La estimación en 0 es el origen del modelo de investigación y las estimaciones en $k$ hace referencia a la utilización infinita que se puede manejar para variables explicativas

$$
\mu_{i}=0
$$


Hace referencia a la no existencia de heterogeneidad, acompañada de $u_{i t}$ que satisface todos los supuestos para el modelo de regresión con los datos panel y la mezcla entre bases de datos para el estudio.

Por lo tanto;

$$
\begin{aligned}
& \qquad K N_{i t}=\beta_{0}+X_{k, i t} \beta_{1}+X_{k, i t} \beta_{2}+\cdots+X_{n, n t} \beta_{n}+u_{i t}(1.2) \\
& K N_{i t}=\beta_{0}+V E_{k, 2013-2019}+V A_{k, 2013-2019}+u_{2013-2019} \\
& \text { Fuente: Elaboración Propia en base a información de (Gujarati y Porter, 2009) }
\end{aligned}
$$

$\mathrm{KN}=$ Capital natural agua

$\mathrm{VE}=$ Variables empresariales

$\vee A=$ Variables ambientales

\subsection{Identificación y descripción de las variables a utilizar}

La información tanto teórica como estadística se obtuvo mediante una búsqueda exhaustiva a través de Internet constituida por: páginas web, bases de datos, investigaciones, informes y artículos. En cuanto a la información estadística se utilizaron distintas entidades responsables de la difusión de información, tales como:

- Consejo privado de competitividad - CPC: Dicha institución posee información anual con respecto al informe de competitividad departamental. Los cuales poseen información sobre activos naturales, gestión ambiental, gestión de riesgo, dinámica empresarial y tamaño del mercado tanto externo como interno (CPC, 2019).

- Sistema de Información Ambiental de Colombia - SIAC: mediante el uso de este sistema se obtuvieron cifras y estadísticas ambientales con respecto a las afectaciones del suelo ocasionadas por las empresas colombianas (SIAC, 2019).

- Comisión Económica para América Latina y el Caribe - CEPAL: Dicha comisión recolecta información con respecto a las empresas certificadas en la norma ISO 14001(CEPALSTAT, 2019).

- Confecámaras: A través de dicho organismo de carácter nacional fue posible la recolección de información en cuanto a la cantidad de empresas totales presentes en Colombia por departamento, sin hacer distinción alguna en el tipo de empresa o el tipo de actividad que estas manejaban (Confecámaras, 2020).

A continuación, se presentan las variables a utilizar dentro de la presente investigación con su respectiva descripción: 
Tabla 1. Variable dependiente

\begin{tabular}{|l|l|l|}
\hline ABREVIATURA & NOMBRE & \multicolumn{1}{|c|}{ EXPLICACIÓN VARIABLE } \\
\hline Cla & Calidad de agua & Es una relación directa con el capital natural \\
& & agua, y hace referencia a diferentes \\
& & características del agua como físicas, químicas, \\
& & biológicas y demás, lo cual permite determinar el \\
& & estado del agua. (Entre más cercano el Dpto. a \\
& & 1,00 mejor es la variable). \\
\hline
\end{tabular}

Fuente: Elaboración propia. Con datos CPC 2019

Tabla 2. Variables Explicativas Empresariales

\begin{tabular}{|c|c|c|}
\hline ABREVIATURA & NOMBRE & EXPLICACIÓN VARIABLE \\
\hline Ced & $\begin{array}{l}\text { Competitividad } \\
\text { empresarial } \\
\text { departamental }\end{array}$ & $\begin{array}{l}\text { Es la relación directa de la empresa con el } \\
\text { departamento, esta encargada de explicar } \\
\text { porcentualmente el desempeño productivo - } \\
\text { económico de las empresas en cada } \\
\text { departamento (Entre mayor sea el porcentaje } \\
\text { en la variable, mejor es el rendimiento). }\end{array}$ \\
\hline Tmi & $\begin{array}{l}\text { Tamaño del } \\
\text { mercado interno }\end{array}$ & $\begin{array}{l}\text { Es la dinámica económica de los mercados } \\
\text { nacionales, se trata de la trazabilidad con } \\
\text { respecto a la oferta y demanda de cada uno de } \\
\text { los mercados productivos y su alcance } \\
\text { económico (entre mayor sea el porcentaje, } \\
\text { mayor es el alcance de las empresas a nivel } \\
\text { nacional). }\end{array}$ \\
\hline Dmg & $\begin{array}{l}\text { Participación de } \\
\text { medianas y } \\
\text { grandes empresas }\end{array}$ & $\begin{array}{l}\text { Se trata de la cantidad de empresas grandes y } \\
\text { medianas que participan en los mercados del } \\
\text { país, incluyendo si cuentan con certificación } \\
\text { ISO } 14001 \text { o no (Un porcentaje mayor indica, } \\
\text { una capacidad amplia de empresas de tamaño } \\
\text { mediano y grande). }\end{array}$ \\
\hline Dpe & $\begin{array}{l}\text { Participación de } \\
\text { pequeñas } \\
\text { empresas }\end{array}$ & $\begin{array}{l}\text { Hace referencia a la cantidad de empresas } \\
\text { pequeñas con y sin certificación ISO } 14001 \text { que } \\
\text { se encuentran funcionando en el mercado } \\
\text { económico (a mayor numero en el porcentaje, } \\
\text { se refiere un mayor número de empresas de } \\
\text { tamaño pequeño). }\end{array}$ \\
\hline Ntotisoc & $\begin{array}{l}\text { Total de empresas } \\
\text { que sin } \\
\text { certificación ISO } \\
14001 \text { a nivel } \\
\text { nacional }\end{array}$ & $\begin{array}{l}\text { Es la cantidad de empresas sin certificación ISO } \\
\text { 14001, en agrupación a los departamentos de } \\
\text { Colombia para los años de estudio. }\end{array}$ \\
\hline Totiso & $\begin{array}{l}\text { Total de empresas } \\
\text { con ISO } 14001 \mathrm{~A} \\
\text { nivel nacional }\end{array}$ & $\begin{array}{l}\text { Es el total referido de empresas que cuenta con } \\
\text { gestión ambiental, basada en certificación de la } \\
\text { Norma ISO } 14001 \text { en Colombia para los años } \\
\text { de estudio. }\end{array}$ \\
\hline
\end{tabular}

Fuente: Elaboración propia. Con datos CPC 2019, CEPALSTAT 2019 y Confecámaras 2020. 
Tabla 3. Variables explicativas ambientales.

\begin{tabular}{|c|c|c|}
\hline ABREVIATURA & NOMBRE & EXPLICACION VARIABLE \\
\hline Mpsb & $\begin{array}{l}\text { Proporción de } \\
\text { superficie cubierta } \\
\text { por bosque }\end{array}$ & $\begin{array}{l}\text { Es la cantidad de cobertura o superficie cubierta } \\
\text { totalmente por los bosques colombianos, se } \\
\text { mide en la investigación como un punto de } \\
\text { referencia que tiene relación directa con } \\
\text { respecto a la producción empresarial y los } \\
\text { daños que esta puede ocasionar en ellos, que a } \\
\text { su vez es representado en la tasa de } \\
\text { deforestación, a favor de la industria. }\end{array}$ \\
\hline Mtds2 & $\begin{array}{l}\text { Tasa de afectación } \\
\text { de desastres } \\
\text { naturales }\end{array}$ & $\begin{array}{l}\text { Es el total de la población porcentualmente } \\
\text { afectada por la destrucción y deterioración al } \\
\text { medio natural y conforma también una relación } \\
\text { directa con el capital natural y sus } \\
\text { componentes, debido a que el deterioro } \\
\text { provoca la existencia de esta variable y esta se } \\
\text { encarga de reflejar un daño a los componentes } \\
\text { del capital natural. }\end{array}$ \\
\hline Afec & $\begin{array}{l}\text { Consecuencias del } \\
\text { deterioro } \\
\text { ambiental en el } \\
\text { capital natural } \\
\text { bosque }\end{array}$ & $\begin{array}{l}\text { Hace referencia al cúmulo de consecuencias } \\
\text { que genera la mala gestión empresarial, } \\
\text { incluyendo altos índices de deforestación, mal } \\
\text { uso de residuos sólidos, mal manejo de áreas } \\
\text { protegidas, Suelos con erosión severa y/o muy } \\
\text { severa y desastres naturales en zonas } \\
\text { vulnerables. }\end{array}$ \\
\hline Mdar & $\begin{array}{l}\text { Disposición } \\
\text { adecuada de } \\
\text { residuos sólidos }\end{array}$ & $\begin{array}{l}\text { Representa la utilización adecuada de los } \\
\text { recursos sólidos por parte de los } \\
\text { departamentos, resultado de la producción } \\
\text { empresarial o industrial. La buena utilización de } \\
\text { dichos recursos, contribuyen a ser una ventaja } \\
\text { de la certificación de las normas de gestión } \\
\text { ambiental. }\end{array}$ \\
\hline
\end{tabular}

Fuente: Elaboración propia. Con datos CPC 2019 y SIAC 2019.

\subsection{Operación del modelo como metodología}

\section{Identificación de la mejor variable dependiente}

Inicialmente para la elaboración de un modelo con datos panel, se corrió un modelo Lasso dado que este posee efectos limpiadores de colinealidad, además de que este logra estabilización de estimaciones y predicciones mediante la contracción de coeficientes (Hoerl y Kennard, 1970). Para ello, se debe identificar las variables que cuentan con valores perdidos y eliminarlos para no provocar ningún daño en el modelo (Carrasco, 2016). De acuerdo con la agrupación de resultados, la variable Cla se escoge como la dependiente del modelo ya que es la variable que mejor es explicada por las variables independientes. 


\section{Identificación del mejor modelo Test de hausman}

Trabajar con datos panel implica la utilización o identificación de los datos de manera grupal o individual, posteriormente se debe saber si el conjunto de datos es explicado por los efectos fijos o aleatorios, entonces se realiza el test de Hausman buscando el mejor efecto;

$\mathrm{Ho}=$ No existe diferencia sistemática entre los coeficientes y se determina una hipótesis nula de no correlación, por lo que se debe utilizar el modelo de efectos fijos. (Probit $>c h i^{2}$ debe ser menor a 0,05)

$\mathrm{Ha}=$ Existe diferencia sistémica entre los coeficientes, debe utilizarse el modelo de efectos aleatorios. (Probit $>c h i^{2}$ debe ser mayor a 0,05)

Para la ejecución del test se debe correr los dos modelos, uno por efectos fijos y otro por efectos aleatorios. El modelo de efectos fijos permite una estimación en intragrupos, donde se busca que el efecto individual del modelo funcione de manera colectiva con las variables explicativas, a su vez permite la conjugación de las variables determinadas y asi hallar la significancia de las más importantes para proceder al desarrollo del modelo a desempeñar. Por otro lado, el modelo de efectos aleatorios es una ampliación de los mínimos cuadrados ordinarios y permite estimar el estudio de los efectos individuales de manera separada a los resultados obtenidos por las variables explicativas. La ejecución de los efectos aleatorios presenta el mismo objetivo que pretende mostrar los efectos fijos, sin embargo, este se diferencia en que la proporción de los efectos conjuntos provienen de los efectos individuales (Labra y Torresillas, 2014).

Tabla 4. Modelo Test de Hausman

\begin{tabular}{|c|c|c|c|c|}
\hline & \multicolumn{2}{|c|}{---- Coefficients ---- } & & \multirow[b]{2}{*}{$\begin{array}{c}\operatorname{Sqrt}\left(\operatorname{diag}\left(V_{-} b-V_{-} B\right)\right) \\
\text { S.E. }\end{array}$} \\
\hline & $\begin{array}{l}\text { (b) } \\
\mathrm{FE}\end{array}$ & $\begin{array}{l}\text { (B) } \\
R E\end{array}$ & $\begin{array}{c}(b-B) \\
\text { Difference }\end{array}$ & \\
\hline Ced &,- 0118469 &,- 0118469 & 0 & 0 \\
\hline Tmi &,- 149426 &,- 149426 & 0 & 0 \\
\hline Dmg & $-6,564685$ & $-6,564685$ & 0 & 0 \\
\hline Dpe & $-6,822484$ & $-6,822484$ & 0 & 0 \\
\hline Mpsb & ,201251 & ,201251 & 0 & 0 \\
\hline Totiso &, 000046 &, 000046 & 0 & 0 \\
\hline Ntotisoc & $-1,71 e-07$ & $-1,71 e-07$ & 0 & 0 \\
\hline Mtds2 &,- 0701495 &,- 0701495 & 0 & 0 \\
\hline \multirow{2}{*}{\multicolumn{5}{|c|}{ Test: Ho: difference in coefficients not systematic }} \\
\hline & & & & \\
\hline \multicolumn{5}{|c|}{$\operatorname{chi} 2(0)=(b-B)^{\prime}\left[\left(V_{-} b-V \_B\right)^{\wedge}(-1)\right](b-B)$} \\
\hline \multicolumn{5}{|c|}{$=0,00$} \\
\hline \multicolumn{5}{|c|}{ Prob $>$ chi2 $=$} \\
\hline
\end{tabular}

Fuente: Estimaciones de los autores mediante el programa Stata. 
Se puede evidenciar con la modelación general, que el mejor modelo es el de efectos fijos (Tabla 4), dado que según el test hausman se acepta la hipótesis nula como la no diferencia sistemática entre los coeficientes. Lo que significa que la hipótesis nula comprueba la existencia de no correlación entre los $\alpha_{i}$ y las variables explicativas. El modelo dio un valor de 0,0003 y se debe aceptar la hipótesis Ho (Reyna, 2007).

\section{RESULTADOS}

El método por efectos fijos prob $>F=0,000$ es una buena estrategia para determinar los datos panel, dado que permite que las variables explicativas estudien de manera relacionada la variable dependiente en una unidad determinada del estudio y así se da una explicación de interconexión de componentes. De manera que el siguiente modelo pretende mostrar la relación que presentan las variables empresariales, que permiten entender la gestión organizativa de las empresas colombianas con respecto al capital natural agua mediante la variable dependiente calidad del agua (Cla) en cada departamento de Colombia, como el indicador principal del grupo.

\subsection{Modelo datos panel}

Efectos fijos

$$
\begin{gathered}
\mathrm{Cla}_{2013-2019}=\alpha_{i}+\mathrm{Ced}_{2013-2019}+\mathrm{Tmi}_{2013-2019}+\mathrm{Dmg}_{2013-2019}+ \\
\mathrm{Dpe}_{2013-2019}+\text { Mpsb } \\
\text { Mtds } 2_{2013-2019}+\mu_{2013-2019}
\end{gathered}
$$

\begin{tabular}{|c|c|c|c|c|c|c|}
\hline \multirow{2}{*}{\multicolumn{3}{|c|}{$\begin{array}{l}\text { Fixed-effects (within) regression } \\
\text { Group variable: cod_dpto }\end{array}$}} & & \multicolumn{2}{|c|}{ Number Of obs } & 231 \\
\hline & & & & \multicolumn{2}{|c|}{ Number of groups } & 33 \\
\hline \multicolumn{3}{|c|}{ R-sq: } & & \multicolumn{2}{|c|}{ Obs per group } & \\
\hline & Within & 0,5944 & & & $\min$ & 7 \\
\hline & Between & 0,1075 & & & avg & 7 \\
\hline & overall & 0,1235 & & & $\max$ & 7 \\
\hline \multirow{2}{*}{\multicolumn{3}{|c|}{$\operatorname{Corr}\left(u_{-} \mathrm{i}, \mathrm{Xb}\right)=-0,0207$}} & & \multicolumn{2}{|c|}{$F(4,295)$} & 123,23 \\
\hline & & & & \multicolumn{2}{|c|}{ Prob $>F$} & 0,0000 \\
\hline Cla & Coef. & Std. Err. & $\mathrm{t}$ & $P>|t|$ & [ 95\% conf. & Inteval ] \\
\hline Ced & $-0,0113664$ & 0,0117209 & $-0,97$ & 0,033 & $-0,011754$ & 0,0344862 \\
\hline Tmi & $-0,1490508$ & 0,077292 & $-1,93$ & 0,055 & $-0,301512$ & 0,0034098 \\
\hline Dmg & $-6,61384$ & 1,948104 & $-3,40$ & 0,001 & $-10,45653$ & $-2,77115$ \\
\hline Dpe & $-6,882342$ & 1,933429 & $-3,56$ & 0,000 & $-10,69609$ & $-3,068598$ \\
\hline
\end{tabular}

Fuente: Elaboración propia de los autores con información de Merli y Perazzi (2013)

Tabla 5. Modelo de efectos fijos 


\begin{tabular}{|l|c|c|c|c|c|c|} 
Mpbs & 0,2019 & 0,0746155 & 2,71 & 0,007 & 0,0547187 & 0,3490812 \\
\hline Totiso & 0,0000461 & $8,79 \mathrm{E}-06$ & 5,24 & 0,000 & 0,0000287 & 0,0000634 \\
\hline Ntotisoc & $-1,71 \mathrm{E}-07$ & $2,85 \mathrm{E}-08$ & $-6,00$ & 0,000 & $-2,27 \mathrm{E}-07$ & $-1,15 \mathrm{E}-07$ \\
\hline Mtds2 & $-0,0710283$ & 0,0263812 & $-2,69$ & 0,008 & $-0,123066$ & $-0,018991$ \\
\hline CCons & 7,201165 & 1,930215 & 3,73 & 0,000 & 3,393762 & 11,00857 \\
\hline sigma_u & 0,14652862 & Prob > F $=0,0000$ \\
\hline sigma_e & 0,02495874 \\
\hline Rho & 0,97180453 & (fraction of variance due to u_i) \\
\hline
\end{tabular}

Fuente: Estimaciones de los autores mediante el programa Stata.

Con respecto al análisis del modelo anterior (Tabla 5) se observa que los modelos por datos panel y series de tiempo cuentan con tres R-squared, siendo estos; Roverall, R-between y R-within, en donde cada uno de ellos está indicado para un tipo de efecto en específico. En nuestro caso, al ser un modelo por efectos fijos, el R-correspondiente a este tipo de modelo vendría siendo el $R^{2}$-within, mientras que los otros $R^{2}$ son conocidos como correlaciones al cuadrado o $R^{2}$ de la segunda ronda de regresiones. De acuerdo con lo anterior, la estimación es de 0,5944 lo que indica que pasado el 0,50 el modelo adquiere precisión. Por otro lado, siguiendo la verificación del modelo, la Prob > F =0,000 indica la significancia conjunta del modelo, mostrando que los coeficientes son diferentes de cero y que el modelo está bien (Reyna, 2007).

En lo que respecta a la significancia individual de las variables $(P>|t|)$, se obtiene de manera general que todas las variables poseen un valor $<0,05$ lo que indica que las variables explicativas tienen una influencia significativa en la variable dependiente (Mayorga y Muñoz, 2000). De tal manera, se da apertura al análisis individual con la comprensión empresarial, liderada por las variables econométricas Ced4, Tmi5, Dmg6 y Dpe7. Por tal razón, a mayor impacto de las variables anteriormente mencionadas, mayor es el deterioro ambiental del capital natural, entonces, con una significancia de 0,033 y un coeficiente negativo de 0,0113664 la competitividad empresarial deja como resultado, que un incremento en esta implica una afectación al capital natural agua y posterior a su calidad, dado que las empresas colombianas se enfatizan en lo mencionado por Chamorro (como se citó en Cerda, 2003) considerando el equilibrio económico y ambiental como un proceso de altos costos que solo producen pérdida de competitividad y mercados extranjeros.

\footnotetext{
${ }^{4}$ Competitividad empresarial departamental

${ }^{5}$ Tamaño del mercado interno

${ }^{6}$ Participación de medianas y grandes empresas

${ }^{7}$ Participación de pequeñas empresas
} 
Por otro lado, lo mismo ocurre con la participación de pequeñas empresas y la participación de medianas y grandes empresas, las cuales respectivamente presentan significancia de 0,000 y 0,001 y coeficientes negativos de 6,882342 y 6,61384 lo que indica que a mayor crecimiento porcentual de estas empresas en el país mayor es el daño a la calidad del agua, tal como planteaba Leff (1999) quien consideraba que el crecimiento económico de la humanidad representado por la cantidad de empresas se alimenta de la pérdida de productividad de los ecosistemas. De esta manera dicho autor establecía que las empresas a través de sus procesos productivos se han encargado de arrasar con el medio ambiente, entonces un aumento en la cantidad de empresas solo empeoraría la situación. A su vez, Mill planteaba que el capital natural como lo es la calidad del agua, es considerado una forma diferente de riqueza y establecía que es preferible conservar la tierra y sus recursos que explotar estos hasta su agotamiento, a través de acciones de carácter empresarial o un aumento en la cantidad de empresas que termina generando múltiples afectaciones ambientales tales como la deforestación, la erosión de los suelos, el calentamiento global (Reynaldo, 2012). Continuando con la parte final que compone la explicación de la empresa, las variables tamaño interno de mercado (Tmi) y la cantidad de empresas sin certificación ambiental (Ntotisoc) destacan una significancia directa con la variable dependiente de 0,055 y 0,000 y coeficientes negativos de 0,1490508 y 1,71e-07, donde se explica que a mayor tamaño empresarial sin procesos de gestión ambiental, se desarrollan técnicas menos amigables con el ambiente y de la misma forma un alcance mayor en el mercado interno incurre en costos más altos que no se pretenden utilizar para procesos de gestión ambiental, dado que según Moreno (2019) a mayor dinámica productiva en el sector empresarial, mayores son sus impactos negativos, dado que le restan importancia sustancial al ambiente por el alcance de su producción.

Para finalizar, las variables de componente ambiental están constituidas por Mpbs8, Mtds29 y Totiso10. La primera de ellas cuenta con una significancia del 0,007 y es la variable proporción de superficie cubierta por bosque, la cual da una representación positiva que se presenta como una relación internamente proporcional, dado que, a mayor proporción de superficie boscosa por parte del cuidado y la conservación de los departamentos en representación de los procesos productivos de la empresa, se da una clase de beneficio para el capital natural agua, con un coeficiente que representa un impacto positivo de 0,2019 en la calidad del agua. Esto se debe a que los bosques y el agua están estrechamente relacionados, debido a que los bosques son una especie de filtro natural que minimiza la erosión local del suelo ya sea ocasionada de manera natural o generada por actividades extractivas; también disminuye los sedimentos

\footnotetext{
${ }^{8}$ Proporción de superficie cubierta por bosque

${ }^{9}$ Tasa de afectación de desastres naturales

${ }^{10}$ Total de empresas con ISO 14001 a nivel nacional
} 
presentes en las masas de agua y filtra otros tipos de contaminantes presentes en el agua (Hamilton, 2009).

De otra manera, la tasa de afectación de desastres naturales (Mtds2) posee una significancia del 0,008 indicando una relación explicativa con la calidad del agua, con un coeficiente negativo de 0,0710283, lo que indica que un alto crecimiento porcentual de los desastres naturales produce agentes contaminantes en el agua y posteriormente inciden en peligros a la salud humana. Como se pudo observar en los resultados de las variables empresariales, las empresas son agentes contaminantes liderados por sus producciones no amigables con el ambiente y los desastres naturales son ocasionados por la contaminación y el deterioro ambiental, es decir, como menciona Chiroles y Gonzáles (2010) los desastres se clasifican entre naturales y aquellos ocasionados por el hombre y su desarrollo industrial, el cual comprende una relación entre sí, lo que a la final termina ocasionando riesgos microbiológicos y un desistimiento en la calidad del agua. A Grosso modo, la variable indica que a mayor crecimiento de estos desastres menor es la calidad del agua.

Finalmente, se tiene que nuestra variable más importante siendo esta el total de empresas con certificación ISO 14001 a nivel nacional (Totiso), posee una significancia de 0,000 que indica la importancia de dicha variable dentro del modelo. Por otro lado, se cuenta con un coeficiente de ponderación positiva de 0,0000461, lo que comprueba que la norma ISO 14001 si ayuda a mitigar los efectos negativos causados por las empresas sobre la calidad del agua. Tal como se ha podido evidenciar mediante investigaciones como la de Enríquez (2009), Páez (2017) y Vargas (2019) en donde un conjunto de distintas empresas adoptó la norma ISO 14001 dado que sus procesos productivos no eran lo suficientemente eficientes y en últimas terminaban contaminando fuentes hídricas. De manera que mediante la norma ISO 14001 se pudieron crear distintos proyectos de gestión ambiental como lo es el control de olores y vertidos, una adecuada gestión de tratamiento de residuos sólidos, etc. Sin embargo, es un coeficiente algo bajo para el tipo de resultados esperados, lo que se debe a la escasa cantidad de empresas que tienen esta norma en sus sistemas de gestión de calidad en el país.

\subsection{Contrastes de especificación}

Tabla 6. Análisis de homocedasticidad

\begin{tabular}{|l|l|ll|r|}
\hline \multicolumn{2}{|c|}{ Cross-sectional time-series FGLS regression } & Number Of obs $=$ & 231 \\
\hline Coefficients: & generalized least squares & Number of groups $=$ & 33 \\
\hline Panels: & homoskedastic & Time periods & $=$ \\
\hline Correlation: & no autocorrelation & Wald chi2 $(8)$ & $=$ & 39,000 \\
\hline Estimated covariances $=$ & 1 & Prob $>$ chi2 & $=$ & 0,0000 \\
\hline Estimated autocorrelations $=$ & 0 & 9 & &
\end{tabular}




\begin{abstract}
Log likelihood
Fuente: Estimaciones de los autores mediante el programa Stata.

El modelo no contiene homocedasticidad (Prob>chi2 = 0,000), ni Autocorrelación, por lo que el panel de datos tiene una buena bondad de ajuste.
\end{abstract}

Tabla 7. Análisis de correlación

\begin{tabular}{|c|c|c|c|}
\hline \multicolumn{4}{|c|}{ Linear regression, correlated panels corrected standard errors (PCSEs) } \\
\hline Group variable: & cod_dpto & Number Of obs & 231 \\
\hline Time variable: & Año & Number of groups $=$ & 33 \\
\hline Panels: & correlated (balanced) & Obs per group & \\
\hline \multirow[t]{2}{*}{ Autocorrelation: } & no autocorrelation & $\min$ & 7 \\
\hline & & avg & 7 \\
\hline Estimated covariances & 561 & $\max$ & 7 \\
\hline Estimated autocorrelations $=$ & 0 & R-squared & 0,6351 \\
\hline Estimated coefficients & 9 & Wald chi2(8) & 284,70 \\
\hline & Prob > chi2 & 0,0000 \\
\hline
\end{tabular}

Fuente: Estimaciones de los autores mediante el programa Stata.

El modelo no contiene Autocorrelación (Prob>chi2 = 0,000), tiene una correlación balanceada y no hay variables repetitivas, por lo que el panel de datos está equilibrado y ajustado.

Tabla 8. Análisis de acciones autorregresivas. Perturbaciones AR.

\begin{tabular}{|c|c|c|c|c|c|}
\hline \multirow{2}{*}{\multicolumn{3}{|c|}{$\begin{array}{l}\text { RE GLS regression with } A R(1) \text { disturbances } \\
\text { Group variable: cod_dpto }\end{array}$}} & \multirow{2}{*}{\multicolumn{2}{|c|}{$\begin{array}{l}\text { Number of obs }= \\
\text { Number of groups }=\end{array}$}} & \multirow{2}{*}{$\frac{231}{33}$} \\
\hline & & & & & \\
\hline \multicolumn{3}{|l|}{ R-sq: } & Obs per group & & \\
\hline Within & $=$ & 0,5873 & & $\min =$ & 7 \\
\hline Between & $=$ & 0,1074 & & $\operatorname{avg}=$ & 7 \\
\hline overall & $=$ & 0,1232 & & $\max =$ & 7 \\
\hline \multicolumn{3}{|c|}{$\operatorname{corr}\left(\mathrm{u}_{\mathrm{i}} \mathrm{i}, \mathrm{Xb}\right)=0$ (assumed) } & Wald chi2(9) & $=$ & 138,60 \\
\hline & & & Prob > chi2 & $=$ & 0 \\
\hline
\end{tabular}

Fuente: Estimaciones de los autores mediante el programa Stata.

Mediante el método de análisis autorregresivo que abarca todo el periodo de estudio, se determina por tercera vez, que el modelo está libre de Autocorrelación por lo que todos los datos acotados anteriormente, permiten un análisis correcto y certero de la variable dependiente.

\title{
4.3 Representación gráfica por de departamentos
}

Para el análisis departamental se realizó una georreferenciación, lo que permite a través de un gráfico organizar cierto conjunto de datos en subcategorías hasta 
llegar a un resultado deseado. En este caso, el objetivo era ilustrar los departamentos que mejores condiciones presentaban en los pilares ambientales y empresariales para los años de estudio 2013 a 2019.

Figura 3. Distribución espacial de la cantidad de empresas en Colombia 2019, empresas certificadas y no certificadas en la ISO 14001.

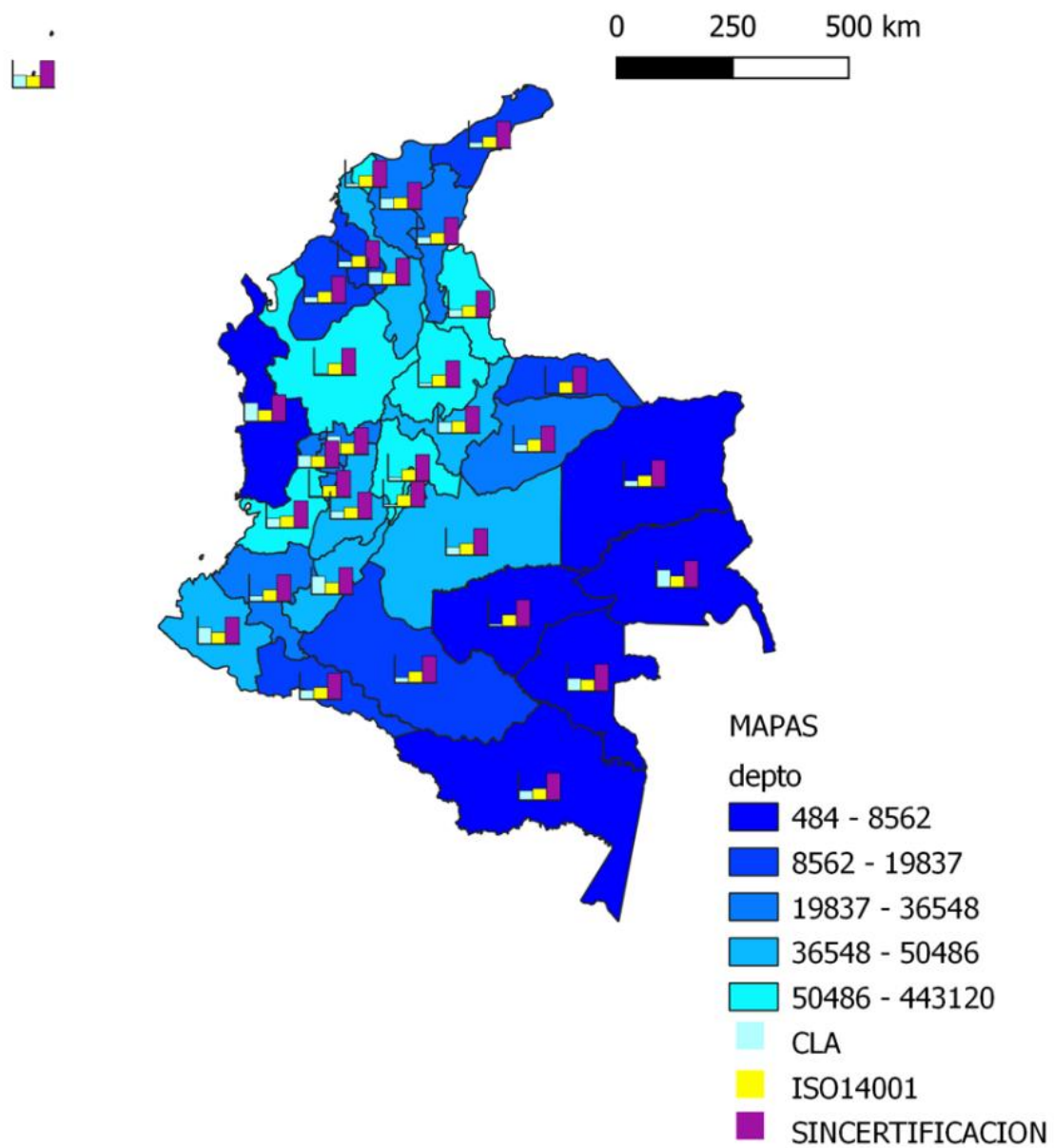

Fuente: Elaboración propia. Con datos CPC 2019.

A partir de la Figura 3, se pueden evidenciar los departamentos con mejor gestión ambiental territorial a partir de la implementación de la Norma ISO 14001, entre los cuales se encuentran Bogotá D.C, Antioquia, Valle del Cauca, Santander, Meta y Cundinamarca aludiendo a la mayor cantidad de empresas certificadas en dicha norma. Esto se debe a que las empresas, por ser de gran tamaño, hacen altas inversiones en sistemas de gestión ambiental y en tecnologías que disminuyen los impactos ambientales. Respecto a la gestión empresarial, los departamentos mencionados son conocidos por ser centros económicos del país además de tener un alto nivel de competitividad y poseer una gran concentración de empresas (Consejo privado de Competitividad y Universidad del Rosario, 2019). Sin embargo, son estos mismos departamentos los que se encuentran entre las zonas que poseen peor calidad de agua en el país, como lo es el caso de Bogotá, 
Antioquia y Cundinamarca que tienen un porcentaje de calidad del agua del 6\%, $4 \%$ y $9 \%$ respectivamente. Esto corresponde a que el impacto generado por las empresas certificadas a pesar de ser positivo es poco significativo, dado que la cantidad de empresas existentes en Colombia sin esta certificación es muchísimo mayor, de manera, que estas contrarrestan el impacto de las empresas que si cuentan con dicho sistema de gestión ambiental.

Figura 4. Distribución espacial de la cantidad de empresas vs componentes ambientales en Colombia, Año 2019.

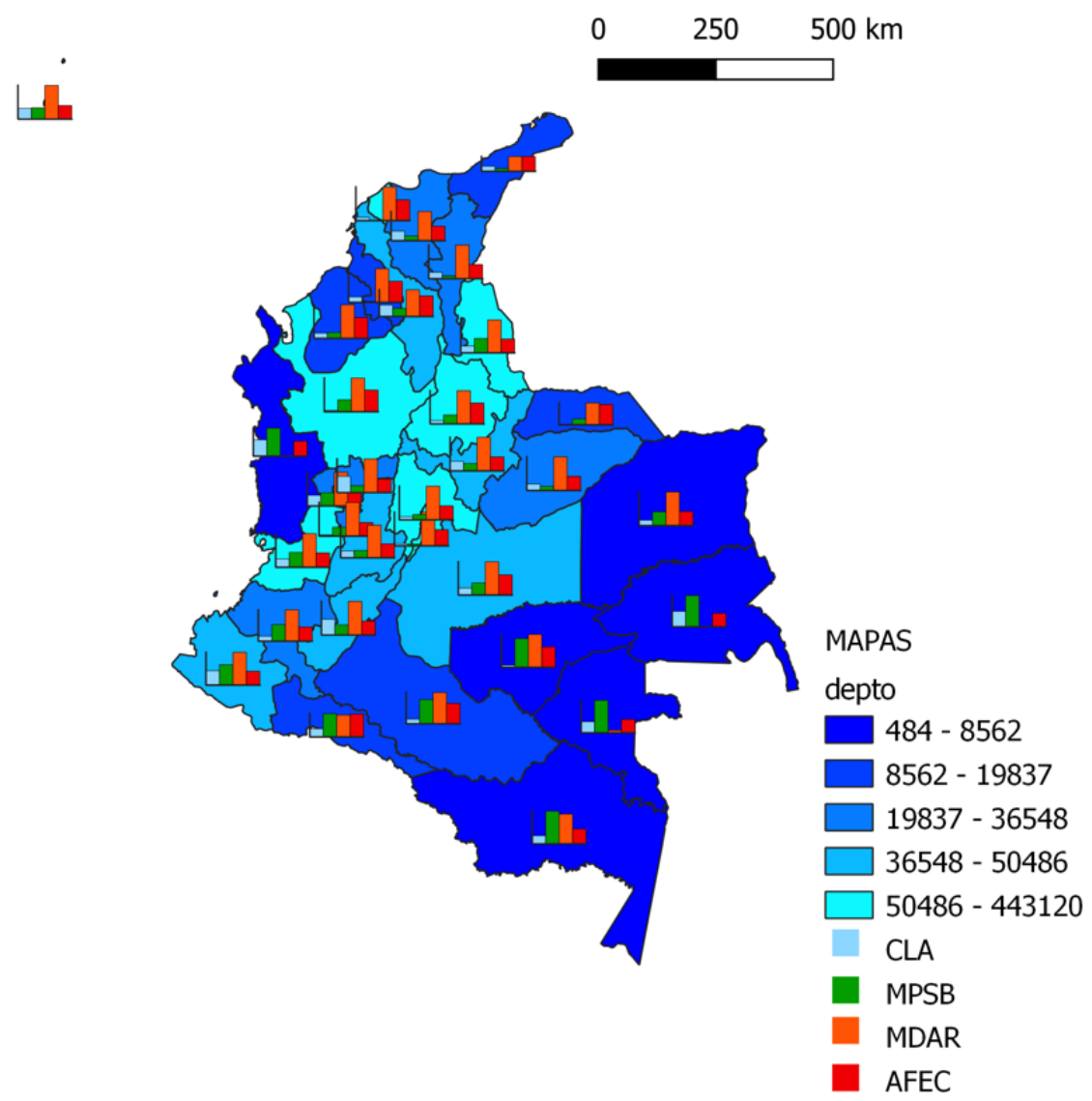

Fuente: Elaboración propia. Con datos CPC 2019.

Por otra parte, en la Figura 4 se observa que el territorio colombiano cuenta con un alto porcentaje de residuos sólidos dispuestos correctamente, según La Superintendencia de Servicios Públicos Domiliarios (SSPD, 2019), el 99,96\% de los departamentos del país tiene mas del 50\% de sus municipios con disposición final autorizada de residuos, lo que determina que una buena adecuación o disposición de estos permitirá mejores resultados para el medio ambiente. Por otro lado, puede evidenciarse en la comparación de estos resultados que las afectaciones, las cuales están contempladas; por desastres naturales, tasa de deforestación y áreas con erosión severa y muy severa, impactan la calidad del 
agua, dado que, a mayor concentración de estas en los departamentos, menor es la calidad del agua, como se puede observar en la Tabla 9.

Tabla 9. Departamentos. Afectaciones VS calidad del agua

\begin{tabular}{|l|l|l|}
\hline \multicolumn{1}{|c|}{ DEPARTAMENTO } & \multicolumn{1}{|c|}{$\begin{array}{c}\text { AFECTACIONES } \\
\text { AMBIENTALES }\end{array}$} & \multicolumn{1}{c|}{ CALIDAD DEL AGUA } \\
\hline Antioquia & $64 \%$ & $4,1 \%$ \\
\hline Arauca & $60 \%$ & $1,7 \%$ \\
\hline Atlántico & $61 \%$ & $9,8 \%$ \\
\hline Caquetá & $60,8 \%$ & $13 \%$ \\
\hline Córdoba & $61,9 \%$ & $15 \%$ \\
\hline Guaviare & $60,6 \%$ & $6,6 \%$ \\
\hline Santander & $62 \%$ & $10,2 \%$ \\
\hline Sucre & $62,3 \%$ & $16 \%$ \\
\hline
\end{tabular}

Fuente: Estimaciones de los autores. Con datos CPC 2019.

Finalmente en zonas como la región amazónica, sus departamentos tienen una poca cantidad de empresas y cuentan con los mejores porcentajes con respecto a la calidad del agua, como lo son Amazonas, Vaupés y Guainía, esto responde a lo que menciona Hamilton (2009), el cual expone que los bosques son un filtro natural para mantener una buena calidad y respectivamente los departamento mencionados cuentan con; 97\%; 96\% y 92\% de superficie cubierta por bosque, ayudando a la poca competitividad empresarial de las zonas. Lo que concluye que, a menor densidad empresarial, mejor es el comportamiento ambiental de los departamentos.

\section{CONCLUSIONES Y RECOMENDACIONES}

La norma ISO 14001 es un sistema de gestión ambiental implementado en el mundo como un sistema para mejorar el impacto de las empresas con respecto al medio ambiente. Mediante la presente investigación se encontraron impactos positivos con respecto a esta norma para la conservación del capital natural agua en Colombia, en donde las empresas certificadas a través de la mejora de sus procesos productivos han disminuido sus niveles de contaminación. Sin embargo, dicho impacto por parte de las empresas es poco significativo por la poca cantidad de empresas con dicha certificación.

Respecto a la gestión empresarial, se tiene que las empresas no certificadas atentan contra la conservación del capital natural a través de procesos organizativos y funcionales que no cuentan con un sistema de gestión ambiental. También, son estas mismas las que ocasionan que las afectaciones ambientales sean cada vez mayores. A su vez, es importante recalcar otros factores empresariales tales como la competitividad empresarial, el tamaño del mercado, la participación de grandes, medianas y pequeñas empresas que terminan agravando la situación, dado que un aumento en la cantidad de empresas o un aumento en la competitividad de las empresas generara una mayor producción, 
una mayor demanda de recursos naturales y una mayor destrucción de este medio.

Por otro lado, se tiene que las variables ambientales tales como la proporción de superficie cubierta por bosque y la tasa de afectación de desastres naturales poseen una relación directa con la calidad del agua, ya que los bosques se consideran según el centro para la Investigación Forestal Internacional (CIFOR, 2013) fuentes de filtro y almacenamiento de agua gracias a su capacidad de absorber lluvia durante estaciones húmedas, siendo capaz de suministrar alrededor del 75\% del agua utilizable en el mundo. Por otro lado, los desastres naturales tienen una relación directa con la contaminación del agua, en donde vemos que uno de los factores principales de la contaminación ambiental es consecuencia de aquellas empresas que no cuentan con gestión ambiental, dado que traen consigo desastres ambientales que generan riesgos microbiológicos que incluso afectan la vida humana (Chiroles y González, 2010).

Finalmente, con la georreferenciación, se pudo obtener una comparación de las diferentes variables que acompañan el objetivo de este estudio, como lo son la calidad del agua con respecto a las empresas certificadas, dado que se comprobó que en los departamentos con mayor certificación ISO 14001 a su vez eran parte de las zonas con peor calidad del agua, debido a que era mayor la cantidad de empresas con procesos no amigables con el ambiente. Sucesivamente, los departamentos con mayor superficie cubierta por bosque, una mejor disposición de residuos sólidos y menor cantidad de empresas, presentaron mayor calidad del agua y una cantidad promedio de certificación ambiental ISO 14001.

Con respecto a las recomendaciones se tiene que, la gestión ambiental territorial, dada la importancia de las instituciones y de la política pública debe definir nuevos marcos jurídicos e instrumentos de gestión ambiental y hacer un mejor uso de los ya existentes. Por otra parte, debe haber un correcto seguimiento, cumplimiento y sanción de estos con el fin de dar pasó a una buena gestión ambiental en el país y que muchas empresas se certifiquen y presenten en sus sistemas funcionalidad organizativa en pro del medio ambiente.

Por otro lado, las empresas deben hacer diagnósticos previos con el fin de analizar la afectación ambiental de sus actividades productivas y así poder definir indicadores reales. A partir de allí, deben orientar su política ambiental y la implementación de este tipo de normas de calidad. Ahora bien, se debe buscar otro tipo de mecanismos de gestión ambiental que no tengan costos tan elevados y que resulten de un proceso de conciencia empresarial voluntaria comprometida con el cuidado de la vida en todas sus expresiones.

En cuanto a la academia, esta debe crear estrategias de generación y apropiación del conocimiento al servicio del sector productivo, mediante investigaciones en 
áreas empresariales y ambientales, que permitan producir alternativas sencillas, integrales y pertinentes las cuales mejoren los procesos al interior de la empresa, el territorio y la política pública. Para esto, es necesaria una mayor disponibilidad y claridad de los datos que ofrecen las instituciones, lo que permite la realización de modelos econométricos óptimos.

\section{REFERENCIAS}

Acuña, N., Figueroa, L., y Wilches, M. J. (2017). Influencia de los sistemas de gestión ambiental en las organizaciones ISO 14001: estudio de caso de empresas manufactureras de Barranquilla. Ingeniare. Revista Chilena de Ingeniería, 25(1), 143153. DOI: http://dx.doi.org/10.4067/s0718-33052017000100143

Cardona, M. B. (2020). Estos son los países más biodiversos del mundo. Viajes National Geographic. Recuperado de: https://viajes.nationalgeographic.com.es/a/paises-masbiodiversidad-mundo 15317/1

Carrasco Carrasco, M. (2016). Técnicas de regularización en regresión: implementación y aplicaciones (Tesis de pregrado). Universidad de Sevilla, España. Recuperado de: https://idus.us.es/bitstream/handle/11441/43746/Carrasco\%20Carrasco\%2C\%20Mar\% C3\%ADa\%20TFG.pdf? sequence=1\&isAllowed $=y$

Castillero Mimenza, O. (2017). Los 15 tipos de investigación (y características). Psicología y Mente. Recuperado de: https://psicologiaymente.com/miscelanea/tipos-deinvestigacion

Ceballos Muñoz, F. A., Ramírez Pascuas, J. E., y Salazar Murcia, J. C. (2019). Impacto de la implementación de un sistema de gestión ambiental ISO 14001:2015 en Colombia (Tesis de pregrado). Universidad Santiago de Cali, Cali, Colombia. Recuperado de: https://repository.usc.edu.co/bitstream/20.500.12421/4100/3/IMPACTO\%20DE\%20LA \%20IMPLEMENTACI\%c3\%93N

Cerda U, A. (2003). Empresa, Competitividad y medio ambiente. Panorama Socioeconómico, 26. Recuperado de: https://www.redalyc.org/pdf/399/39902604.pdf CIFOR. (2013). Bosques y agua: lo que deberían saber los formuladores de políticas. Center for International Forestry Research (CIFOR). DOI: https://doi.org/10.17528/cifor/004175

Chiroles Rubalcaba, S., y Gonzáles Gonzáles, M. I. (2010). Seguridad del agua en situaciones de emergencia y desastres. Peligros microbiológicos y su evaluación. Revista Cubana de Higiene y Epidemiología, 48(1), 93-105. Recuperado de: http://scielo.sld.cu/pdf/hie/v48n1/hie10110.pdf

CEPALSTAT. (2019). Empresas con certificación ISO 14001. [Base de datos]. Recuperado de:

https://estadisticas.cepal.org/cepalstat/tabulador/Consultalntegrada.asp?idlndicador= 1763\&idioma $=e$ 
Confecámaras. (2020). Número de empresas por departamento 2013-2019. [Base de datos]. Comunicación Personal.

Consejo privado de Competitividad y Universidad del Rosario. (2019). Índice Departamental de competitividad 2019. Bogotá. Recuperado de: https://compite.com.co/wp-content/uploads/2019/11/CPC IDC 2019 WEB.pdf

CPC. (2019). Índice Departamental de Competitividad. [Base de datos]. Recuperado de: https://idc.compite.com.co

Enríquez Bernal, L. M. (2009). Estrategia para la implementación de la norma iso 14001 en empresas productoras de palma de aceite de la zona oriental colombiana (Tesis de maestría). Pontificia Universidad Javeriana, Bogotá, Colombia. Recuperado de: http://hdl.handle.net/10554/717

Gonzales Herrero, E. (2015). El agua en la industria: crece la demanda frente a un recurso más escaso. iagua. Recuperado de: https://www.iagua.es/noticias/eva-gonzalezherrero/15/07/27/agua-industria-crece-demanda-frente-recurso-mas-escaso

Gujarati, D. N., y Porter, D. C. (2009). Basic Econometrics, Fifth Edition. McGraw Hill Recuperado de: https://cbpbu.ac.in/userfiles/file/2020/STUDY MAT/ECO/1.pdf

Hamilton, L. S. (2009). Los bosques y el agua. Estudio temático elaborado en el ámbito de la Evaluación de los recursos forestales mundiales 2005. Organización de las Naciones Unidas para la Alimentación y la Agricultura (FAO). Recuperado de: http://www.fao.org/3/i0410s/i0410s00.pdf

Hoerl, A. E., y Kennard, R. W. (1970). Ridge regression: biased estimation for Nonorthogonal Problems. Technometrics, 12(1), 55-67. DOl: https://doi.org/10.2307/1267351

IDEAM (2019). Estudio Nacional del Agua 2018. Recuperado de: http://www.andi.com.co/Uploads/ENA 2018-comprimido.pdf

Instituto colombiano de normas técnicas y certificación (ICONTEC). (2015). Sistemas de gestión ambiental. requisitos con orientación para su uso. Recuperado de: https://informacion.unad.edu.co/images/control interno/NTC ISO 14001 2015.pdf

Labra, R., y Torrecillas, C. (2014). Guía CERO para datos de panel. Un enfoque práctico. Cátedra UAM-Accenture en Economía y Gestión de la Innovación. Recuperado de: https://www.catedrauaminnova.com/documents/Working\%20papers/WP2014 16 Gui a\%20CERO\%20para\%20datos\%20de\%20panel Un\%20enfoque\%20practico.pdf

Leff, E. (1999). La insoportable levedad de la globalización. La capitalización de la naturaleza y las estrategias fatales de la sustentabilidad. En C. R. Ruiz Moreno. (Ed.), Desarrollo sustentable irealidad o retorica? (pp. 22-43). Ecuador: ABYA-YALA. Recuperado 
https://digitalrepository.unm.edu/cgi/viewcontent.cgi?article $=1114 \&$ context=abya yala \#page $=22$

Mayorga M, M., \& Muñoz S, E. (2000). La técnica de datos de panel una guía para su uso e interpretación. Banco Central de Costa Rica. Recuperado de: https://repositorioinvestigaciones.bccr.fi.cr/bitstream/handle/20.500.12506/208/200 T ecnica datos panel una guia para su uso e interpretacion.pdf?sequence=1\&isAllow $\underline{e d=y}$

Merli, G. O., \& Perazzi, J. R. (2013). Modelos de regresión de datos panel y su aplicación en la evaluación de impactos de programas sociales. Telos, 15(1), 119-127. Recuperado de: https://www.redalyc.org/pdf/993/99326637008.pdf

Moreno. (2019). El impacto de los procesos industriales en el medio ambiente. CEUPE. Recuperado de: https://www.ceupe.com/blog/impacto-procesos-industriales-medioambiente.html

Otero-Ortega, A. (2018). ENFOQUES DE INVESTIGACIÓN. ResearchGate. Recuperado de: https://www.researchgate.net/publication/326905435 ENFOQUES DE INVESTIGACIO $\underline{\mathrm{N}}$

Páez Velandia, A. (2017). Aportes de la gestión ambiental a un modelo de sostenibilidad empresarial (Tesis de especialización). Fundación Universidad América, Bogotá, Colombia. Recuperado de: https://repository.uamerica.edu.co/bitstream/20.500.11839/7033/1/57234-2017-II$\underline{\text { GA.pdf }}$

Panayotou, T., \& Vincent, J. R. (1999). Regulación del medio ambiente y competitividad. Journal of Economics, Finance and Administrative Science, 5(9-10), 25-45. Recuperado de: https://jefas.esan.edu.pe/index.php/jefas/article/view/101

Reyna, O. T. (2007). Panel Data Analysis Fixed and Random Effects using Stata. Princeton University. Recuperado de: https://www.princeton.edu/ otorres/Panel101.pdf

Reynaldo Argüelles, C. L. (2012). La economía ambiental y su evolución en el pensamiento económico. DELOS: Desarrollo Local Sostenible, 5(13). https://dialnet.unirioja.es/servlet/articulo?codigo $=6421175$

SIAC. (2019). Cifras y estadísticas ambientales, Afectaciones del suelo. [Base de datos]. Recuperado de: http://cifras.siac.gov.co/Portal-SIAC-web/faces/suelo inicio.xhtml

Superintendencia de Servicios Públicos Domiliarios. (2019). Disposición final de residuos sólidos informe nacional- 2018. Recuperado de: https://www.superservicios.gov.co/sites/default/archivos/Publicaciones/Publicaciones/ 2020/Ene/informe nacional disposicion final 2019 1.pdf 
Unesco. (2016). El agua, fuente de empleo y crecimiento económico, según nuevo informe de las Naciones Unidas. Recuperado de: https://es.unesco.org/news/aguafuente-empleo-y-crecimiento-economico-segun-nuevo-informe-naciones-unidas

Vargas Herrera, J. A. (2019). El desarrollo sostenible en Colombia y Brasil: Contribuciones de la implementación de la norma ISO14001 en las organizaciones (Tesis de diplomado). Universidad Militar Nueva Granada, Bogotá, Colombia. Recuperado de: http://hdl.handle.net/10654/32442

Zapata Gómez, A. (2007). La gestión ambiental en el sector empresarial, una visión bajo el enfoque empresa-entorno como estrategia de competitividad (Tesis de Maestría). Universidad Nacional de Colombia, Manizales, Colombia. Recuperado de: https://repositorio.unal.edu.co/handle/unal/2763 